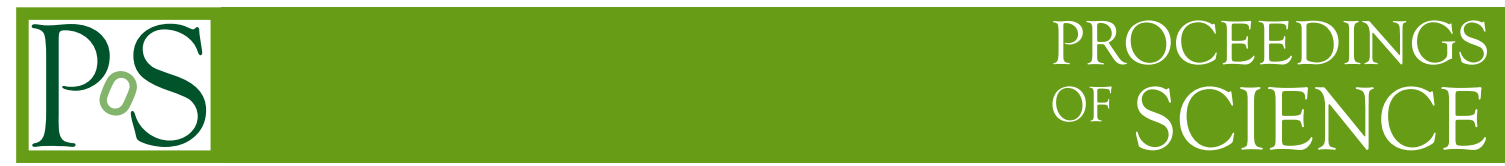

\title{
Static quark free energies at finite temperature
}

\author{
Z. Fodor ${ }^{1,2}$, A. Jakovác ${ }^{2}$, S.D. Katz ${ }^{1,2}$, K.K. Szabó ${ }^{2}$ \\ ${ }^{1}$ Institute for Theoretical Physics, Eötvös University, H-1117 Budapest, Hungary. \\ ${ }^{2}$ Department of Physics, University of Wuppertal, D-42097 Wuppertal, Germany. \\ E-mail: jakovac@esr.phy.bme.hu
}

We determine the static quark free energies around the transition temperature using $2+1$ flavors of staggered fermions. Simulations are carried out on $N_{t}=4,6,8$ and 10 lattices using physical quark masses. The free energies extracted from Polyakov-loop correlators are extrapolated to the continuum limit.

The XXV International Symposium on Lattice Field Theory

July 30-4 August 2007

Regensburg, Germany 


\section{Introduction}

Our aim is to compute the free energy of a static quark-antiquark pair. There are several measurements on this quantity in the literature (for recent publications cf. [1, 2, 3, 4]). Here we go beyond these computations, we use physical quark masses and perform a careful continuum limit extrapolation with the necessary renormalization procedure.

The quark-antiquark free energy can be expressed as correlators of Polyakov loops:

$$
e^{-F_{\bar{q} q}(\mathbf{r}) / T} \sim \sum_{\mathbf{x}}\left\langle\operatorname{Tr} P(\mathbf{x}) \operatorname{Tr} P^{\dagger}(\mathbf{x}+\mathbf{r})\right\rangle,
$$

where $\mathbf{r}$ is a vector in the spacial direction, $T=1 /\left(N_{t} a\right)$ is the temperature and $\mathbf{x}$ runs over all the spatial lattice sites. $P$ is the Polyakov loop

$$
P(\mathbf{x})=\prod_{x_{4}=0}^{N_{t}-1} U_{4}\left(\mathbf{x}, x_{4}\right),
$$

where $U_{\mu}(x) \in S U(3)$ is the link gauge field.

In pure gauge theory we expect that the Polyakov loop correlator behaves Coulomb-like at short distances. In the deconfined phase the Coulomb behavior is screened at large distances, the exponential range defines the screening mass. In the confined phase the free energy is linearly rising, the derivative of the rise is the string tension. This behavior can give an account for the quark confinement and Regge trajectories at zero temperature.

The above picture is modified, however, when we include dynamical quarks. At large distances it is favorable to generate a quark-antiquark pair from the vacuum, which then screens the color field between the two Polyakov loops [5]. From this point (the string breaking scale) the lowest energy level will be insensitive of the position of the heavy quarks, resulting in a constant free energy. The value of this constant restricts the possible bound state energies, calculated in the given potential, as no bound state can be formed with energy larger then the maximum energy.

At finite temperature the above picture persists, but we can also have general expectations about the temperature dependence. Physically we expect that in a thermal vacuum it is easier to generate a quark-antiquark pair than at $T=0$, since there are thermally excited particles around which can scatter on the gluonic string between the static quark-antiquark pair. The gluonic string, being excited itself, can more easily break into a dynamical quark-antiquark pair. This suggests that the string breaking scale and so the flattened free energy value decreases with the temperature. This dynamical picture coincides with the thermodynamical expectation. The negative temperature derivative of the free energy is the entropy, which must be positive in a stable system:

$$
-\left.\frac{\partial F_{\bar{q} q}(\mathbf{r}, T)}{\partial T}\right|_{V}=S_{\bar{q} q}(\mathbf{r}, T)>0 .
$$

This formula should be true for any $\mathbf{r}$, since $\mathbf{r}$ here is just a parameter, telling the position of the fixed Polyakov loops. As a consequence we expect that at any point the quark-antiquark free energy decreases with the temperature. This condition can be an important check for the correctness of the renormalization procedure.

String breaking effects compete with screening. If the free energy is screened before it can rise to the string breaking scale, then screening wins, otherwise the string breaking effect. But the 
main features of the free energy are the same in both cases. Since there is no phase transition in QCD, the two regimes are connected with each other continuously.

\section{Renormalization}

When we approach the continuum limit, the value of the unrenormalized free energy diverges. This is because in a single Polyakov loop the self-energy is divergent. We expect:

$$
\left.\langle\operatorname{Tr} P(x)\rangle\right|_{\mathrm{div}}=e^{-C(a) N_{t} a}=e^{-C(a) / T},
$$

where $C(a) \rightarrow \infty$ in the continuum limit. At finite $a$ the specific value of $C(a)$ has no physical meaning, since it depends on how we define the "divergent part" of the self-energy (renormalization scheme). Although the constant $C(a)$ can be chosen in different ways, it is important that it should only depend on the lattice spacing. In the literature there are several ways to fix this constant $[3,6]$.

Subtracting the divergent part from the free energy, the renormalized free energy can be defined as

$$
e^{-F_{\bar{q} q}^{\mathrm{ren}}(\mathbf{r}, a) / T}=e^{-F_{\bar{q} q}(\mathbf{r}, a) / T} e^{2 C(a) / T}, \quad \Rightarrow \quad F_{\bar{q} q}^{\mathrm{ren}}(\mathbf{r}, a)=F_{\bar{q} q}(\mathbf{r}, a)-2 C(a) .
$$

A possible way of fixing $C(a)$ is to take a physical observable based on $F_{\bar{q} q}$, and requiring that it should be independent of $a$. We emphasize that there is no restriction on the physical quantity other than it must be fixed and be finite if $F_{\bar{q} q}$ is finite. It needs not to be a zero temperature observable. In fact, the most useful quantity in our calculation was the constant value of the free energy after the string breaking/screening, at a fixed temperature. We kept this value 0 for all $a$, that is we have chosen the constant $C(a)$ as

$$
2 C(a)=F_{\bar{q} q}\left(\mathbf{r} \rightarrow \infty, a, T_{0}\right),
$$

with a fixed $T_{0}$ (its value was $T_{0}=190 \mathrm{MeV}$ in the calculation). The renormalized free energy therefore reads at any temperatures as

$$
F_{\bar{q} q}^{\mathrm{ren}}(\mathbf{r}, a, T)=F_{\bar{q} q}(\mathbf{r}, a, T)-F_{\bar{q} q}\left(\mathbf{r} \rightarrow \infty, a, T_{0}\right) .
$$

\section{Results}

We used Symanzik improved gauge- and stout improved staggered fermionic actions. The parameters of the action were the same as in Refs. [7]. Table 1 summarizes the lattices we used for the measurements. These are the same gauge configurations as in Ref [8]. We measured the Polyakov loop correlator for each possible $\mathbf{r}$ values which could fit in the half-size of the spatial extent. Next we averaged the correlator for distances $r=\sqrt{\mathbf{r}^{2}}$, including on- and off-axis contributions. Note, that we take the continuum limit, where rotation invariance should be restored. We binned the data according to the lattice spacing, averaging Polyakov loop correlators with the same $n=($ int $)(r+0.5)$. From the binned Polyakov loops we computed the binned free energy as $F_{\bar{q} q, n}=\ln \langle P P\rangle_{n} /\left(N_{t} a\right)$. The $a(\beta)$ function was taken from the lines of constant physics determined earlier for these set of lattices in [8,9]. There the condition for the determination of $\beta$ and the quark masses was to keep the ratios of the physical values of $m_{\pi}, f_{K}$ and $m_{K}$ fixed at zero temperature. 


\begin{tabular}{|c|c|c|}
\hline geometry & $\beta$ range & \# of $\beta$ values \\
\hline \hline $16^{3} \times 4$ & $3.2-3.425$ & 19 \\
\hline $24^{3} \times 6$ & $3.45-3.705$ & 11 \\
\hline $32^{3} \times 8$ & $3.57-3.725$ & 7 \\
\hline $48 \times 40^{2} \times 10$ & $3.63-3.86$ & 7 \\
\hline
\end{tabular}

Table 1: The lattices used for the Polyakov loop correlators.

The binned free energy was renormalized in the following way. For each $N_{t}$ and $\beta$ we fitted the free energies with the function

$$
F_{f i t}(r)=\frac{a e^{-b r}}{r^{c}}+d
$$

We then interpolated the fitted functions on each $N_{t}$ to the $\beta$ values corresponding to $T_{0}=190 \mathrm{MeV}$. The asymptotic values of these four $\left(N_{t}=4,6,8,10\right)$ free energies gave $2 C$ as a function of $\beta$. The four points and a fitted polynomial can be seen on Fig. 1 . The value of $T_{0}=190 \mathrm{MeV}$ was motivated

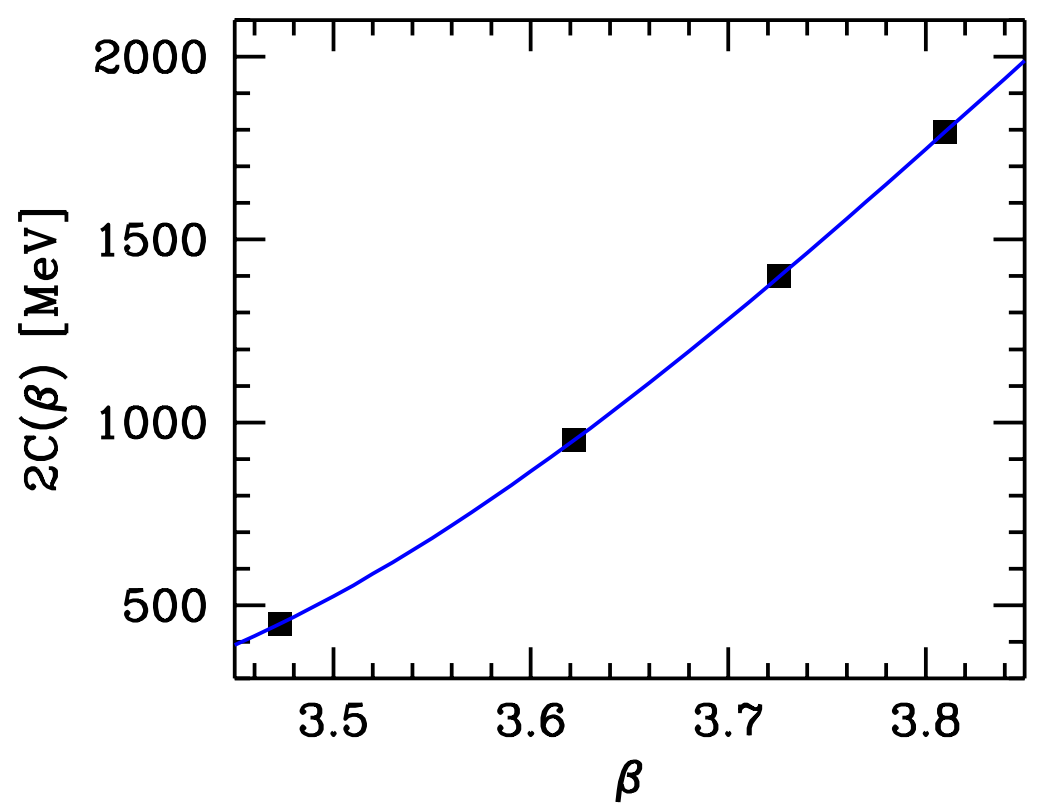

Figure 1: The additive renormalization factor to the free energy, as a function of $\beta$.

by the fact that it lies already in the deconfined phase where the statistical errors of the free energy are much smaller than in the confined phase. At this temperature the free energy at large distances, by definition, has no lattice spacing dependence. At nearby temperatures we expect similarly good behavior.

Once we have the value of $2 C(\beta)$ we can subtract it from all free energy values, thus having their renormalized value. The result for temperature $T=189 \mathrm{MeV}$ can be seen on Fig. 2 together with the fitted curve. This $N_{t}=8$ point was the closest to the renormalization temperature $T_{0}$, where we had raw data without interpolation. 


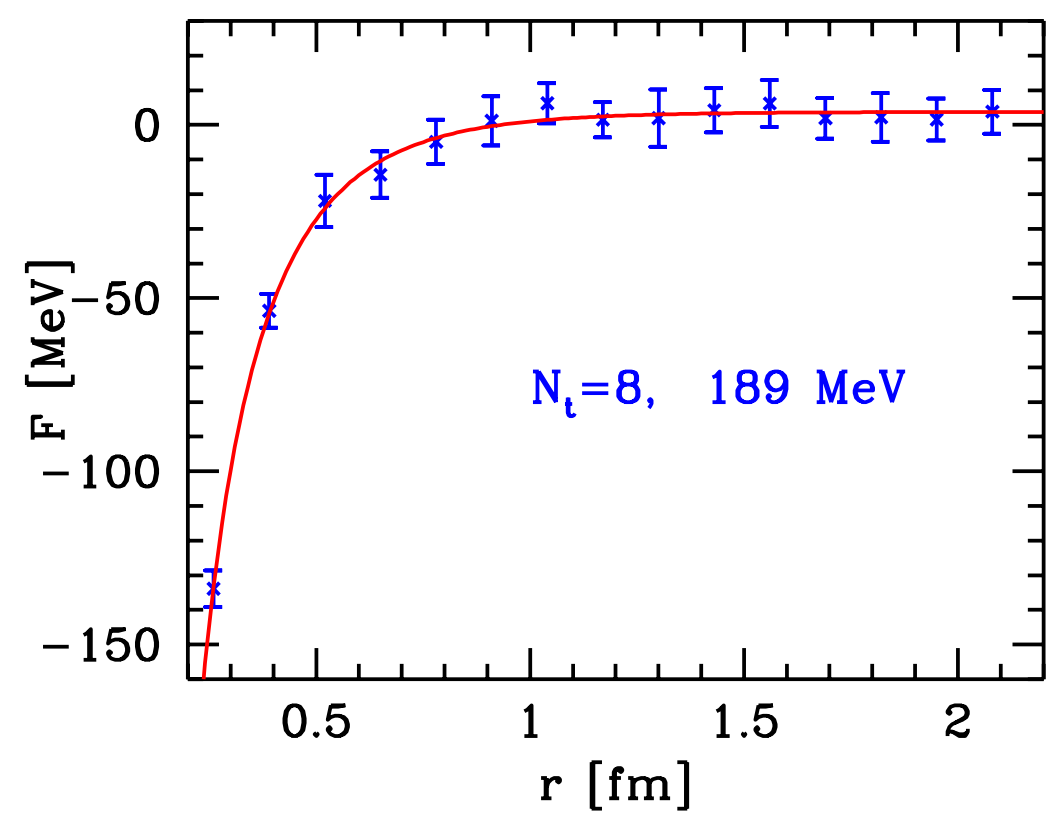

Figure 2: The renormalized free energy at $T=189 \mathrm{MeV}$. The smooth curve is $F_{f i t}$ discussed in the text.

Since we now know the renormalized free energy for all lattice spacings, we can take the continuum limit by using the $N_{t}=4,6,8$ and 10 free energies, and extrapolate in $1 / N_{t}^{2} \sim a^{2} \rightarrow 0$. In Fig. 3 one can see the free energies at different $N_{t}$ values for $T=200 \mathrm{MeV}$. We can see that the lattice artefacts are small, $N_{t}=8$ and 10 results almost completely coincide. Therefore a safe extrapolation to $1 / N_{t}^{2}=0$ is possible. We estimate the systematic error of this extrapolation by comparing the results coming from $N_{t}=6,8,10$ extrapolation and $N_{t}=8,10$ extrapolation. The result for the renormalized free energy at different temperatures, including both the statistical and the systematic errors, can be seen on Fig. 4.

\section{Conclusions}

We have determined the finite temperature renormalized static quark free energy in QCD with dynamical staggered fermions using physical quark masses. According to our expectations, the free energy is Coulomb-like for small distances, at larger distances it is screened and/or exhibits string breaking, and so flattens out. An important feature of the computation was the careful renormalization procedure. We fixed a physical quantity: the asymptotic value of the free energy at $T=190$ $\mathrm{MeV}$, which was kept zero for all lattice spacings. This defines the additive renormalization factor for the quark-antiquark free energy as a function of the lattice spacing. At different temperatures and different distances this factor must be used to renormalize the free energy. The free energy defined in this way is monotonically decreasing as a function of the temperature for all distances.

\section{Acknowledgment}

Partial support of grants of DFG F0 502/1, EU I3HP, OTKA AT049652 and OTKA K68108 


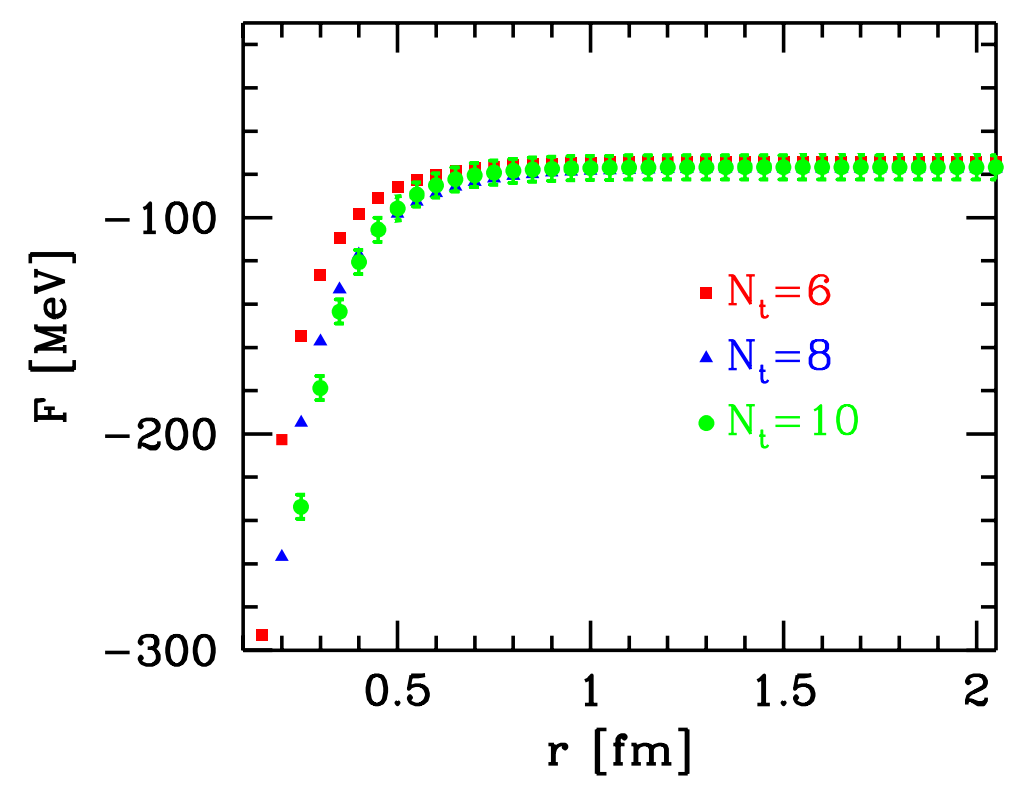

Figure 3: The renormalized free energies for $N_{t}=6,8$ and 10 .

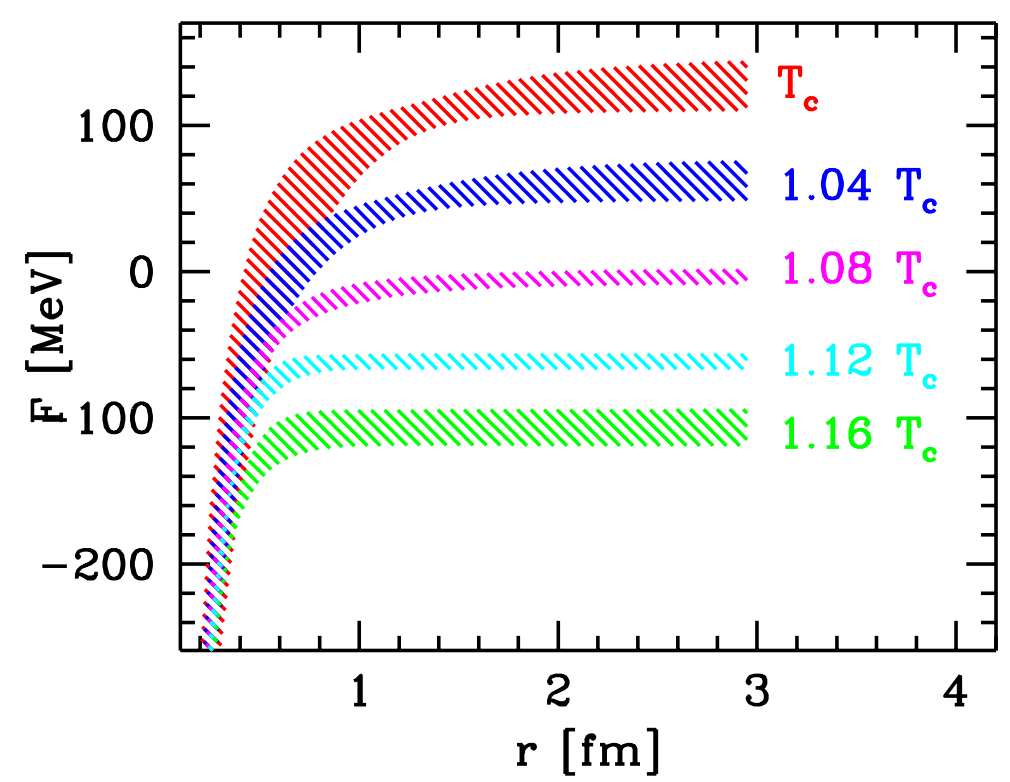

Figure 4: The renormalized free energies in the continuum limit. 
is acknowledged. A.J. is supported by the Humboldt Foundation.

\section{References}

[1] O. Kaczmarek, F. Karsch, P. Petreczky and F. Zantow, Phys. Lett. B 543 (2002) 41 [arXiv:hep-lat/0207002].

[2] V. Bornyakov et al., arXiv:hep-lat/0301002.

[3] O. Kaczmarek, F. Karsch, F. Zantow and P. Petreczky, Phys. Rev. D 70 (2004) 074505 [Erratum-ibid. D 72 (2005) 059903] [arXiv:hep-lat/0406036].

[4] Y. Maezawa, N. Ukita, S. Aoki, S. Ejiri, T. Hatsuda, N. Ishii and K. Kanaya [WHOT-QCD Collaboration], Phys. Rev. D 75 (2007) 074501 [arXiv:hep-lat/0702004].

[5] G. S. Bali, H. Neff, T. Duessel, T. Lippert and K. Schilling [SESAM Collaboration], Phys. Rev. D 71 (2005) 114513 [arXiv:hep-lat/0505012].

[6] Z. Fodor, C. Guse, S. D. Katz and K. K. Szabo, PoS LAT2005 (2006) 178 [arXiv:hep-lat/0511033].

[7] Y. Aoki, Z. Fodor, S. D. Katz and K. K. Szabo, JHEP 0601, 089 (2006) [arXiv:hep-lat/0510084].

[8] Y. Aoki, Z. Fodor, S. D. Katz and K. K. Szabo, Phys. Lett. B 643 (2006) 46 [arXiv:hep-lat/0609068].

[9] Y. Aoki, G. Endrodi, Z. Fodor, S. D. Katz and K. K. Szabo, Nature 443 (2006) 675 [arXiv:hep-lat/0611014]. 